\title{
THE MEASUREMENT OF CARDIAC OUTPUT. AN IMPROVEMENT OF THE ACETYLENE METHOD PROVIDING AN INHERENT CHECK
}

\author{
By WRIGHT ADAMS AND IRENE SANDIFORD \\ (From the Department of Medicine, University of Chicago, Chicago)
}

(Received for publication August 16, 1940)

The method of Marshall and Grollman (1) for measuring the arteriovenous oxygen difference by forcible rebreathing of a mixture of air, oxygen and acetylene has been criticized on the grounds that gas samples are taken during a period when significant amounts of blood containing acetylene are returning to the lungs; that is, from 15 to 23 seconds after the beginning of rebreathing. On the other hand, if samples are taken too soon after the beginning of rebreathing they may contain bag or lung air which has not been completely mixed. The three sample technique (2) which provides for the calculation of duplicate results for arteriovenous difference was introduced to overcome these objections. However, the use of this procedure in 76 experiments in our laboratory yielded second values which averaged 12 per cent higher than the first values, indicating increasing recirculation of acetylene. Hence it is not justifiable to accept even the first value, because without clear-cut checks between the two figures either or both of them may be subject to errors due either to recirculation or to incomplete lung-bag equilibrium. An attempt was made to evaluate these two sources of error and to devise a method which eliminated them.

\section{EXPERIMENTAL}

Multiple expiratory samples. The valve as described by Grollman was modified to permit withdrawal of six samples during successive expirations at the time of forcible rebreathing of the acetylene air mixture. The arteriovenous oxygen difference calculated from successive pairs showed a progressive rise when the samples were taken from 12 to 30 seconds after the beginning of rebreathing. During the first 10 seconds the results were irregular, often very low or very high, and successive values frequently varied widely.

Multiple samples of " alveolar" and "bag" gas. The valve was modified further by the introduc- tion of a quickly acting flap valve between the bag and the sampling tube connections at the mouthpiece and provision was made for sampling at the

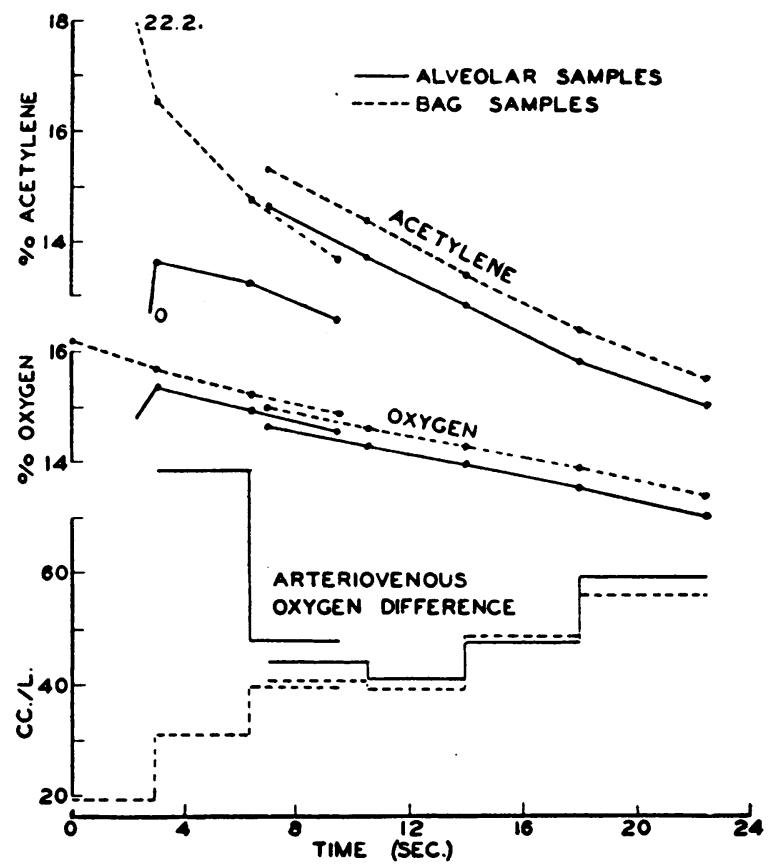

Fig. 1. Acetylene and Oxygen Concentrations in Alveolar and Bag Gases During Two Rebreathings, TOgether With the Corresponding Arteriovenous Oxygen Differences

Samples were taken at the end of successive expirations. The first experiment extended through the first three breaths ( 9.5 seconds). In the second experiment sampling was started following the third breath ( 7 seconds) and carried on for five successive breaths.

other end of the bag as well. At the end of successive expirations the flap valve was closed momentarily and samples composed principally of alveolar gas were taken at the mouthpiece; other samples, representing a mixture of some alveolar gas with all of the gas from the dead spaces in the bag, valve, and upper respiratory tract, were taken simultaneously from the end of the bag. 
ARTERIOVENOUS OXYGEN DIFFERENCE FROM SUCCESSIVE ALVEOLAR AND BAG SAMPLES.
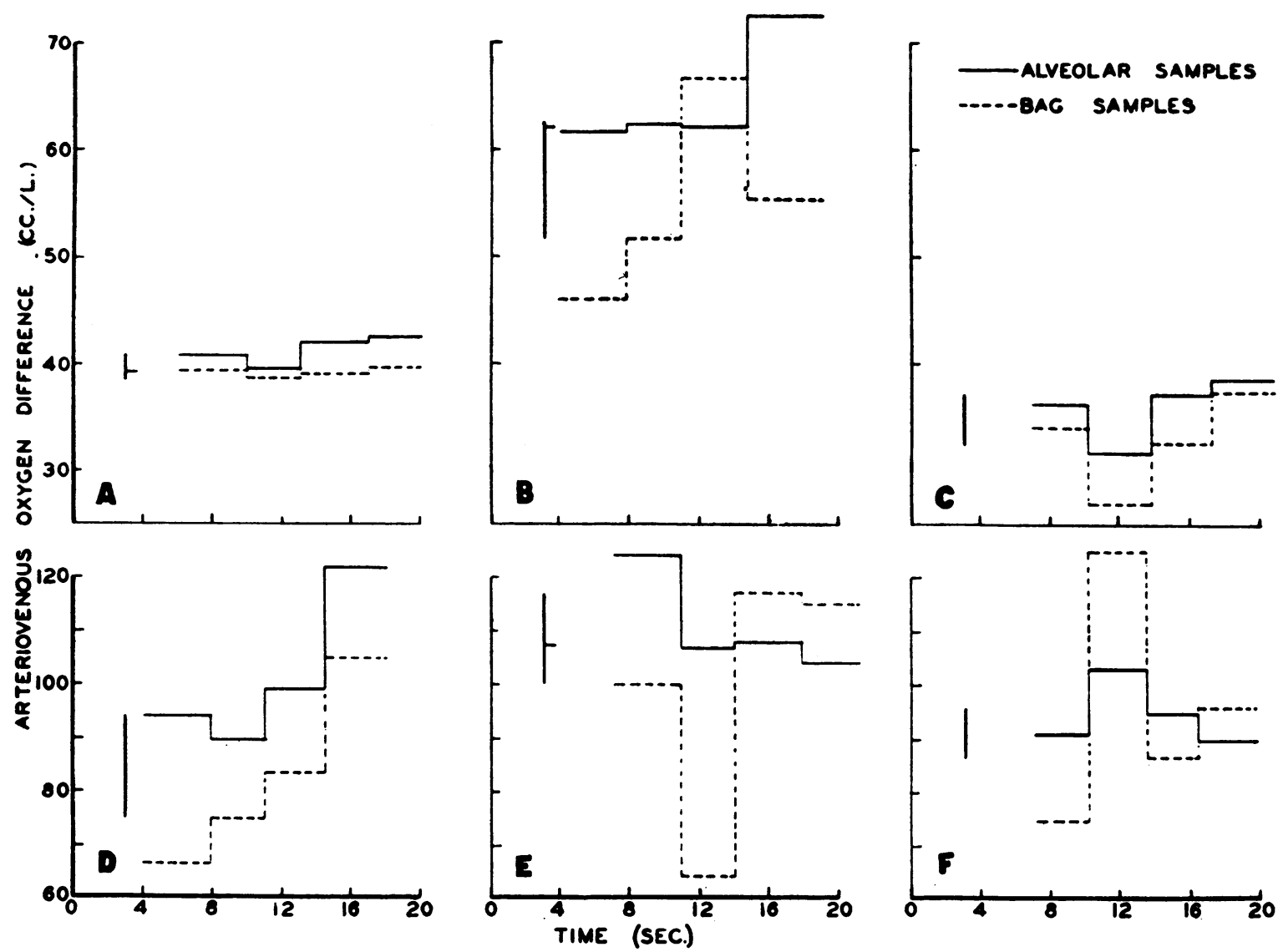

Fig. 2. Graphs of Actual Results of Rebreathing to Illustrate Types of Accurate and Inaccurate Detrrminations

The length of the vertical bar at the left of each figure is the range within which the value for arteriovenous oxygen difference can be assumed to lie. When a probable correct figure can be pointed out within this range, it is indicated by a horizontal line drawn from the vertical bar.

After lung-bag equilibrium had occurred the gas concentrations in successive "alveolar" and "bag" samples fell on approximately parallel lines. This situation is present early in the case of oxygen, since the difference between normal alveolar oxygen concentration and bag oxygen concentration at the beginning of rebreathing is relatively small. In a typical experiment (Figure 1) oxygen equilibrium was established in one breath. Some delay occurred in the case of acetylene, since initially the bag concentration was over 20 per cent and the alveolar concentration was zero. Acetylene equilibrium was established at the fourth breath (Figure 1). In the second rebreathing (Figure 1) sampling was begun after the third breath and both oxygen and acetylene values were found parallel. After the establishment of equilibrium the decrease in acetylene values (acetylene difference) from breath to breath was the same in "alveolar" and "bag" gas; prior to this the decrease was smaller in the former than in the latter.

\section{THE MODIFIED METHOD}

The above-described experiments proving the difficulty in determining when lung-bag equilibrium has been reached with the Grollman technique and the demonstration that this difficulty is obviated by means of the above-described modification of the valve and method, led to the formulation of the present technique for measuring cardiac output by means of the forcible rebreathing of mixtures of air, oxygen, and acetylene.

The gas mixture is made up as described by Marshall 
and Grollman (1) equal in volume to two-thirds to threefourths of the vital capacity of the subject. Strenuous preliminary expiration leaving only the residual air in the lungs, together with a large amount of bag gas, favors rapid equilibration. The rebreathing is performed with the subject in bed elevated by a back rest to about $45^{\circ}$. Sampling is accomplished from both the mouthpiece and the bag, as described above, using the modified valve. Analyses are performed by means of a modified Haldane apparatus and the oxygen consumption is determined in duplicate by means of the Benedict-Roth apparatus.

Plotting the obtained oxygen and acetylene values (Figure 1) does not permit accurate judgments regarding the slope of lines in various periods, since estimation of the time of sampling is subject to an error of at least half a second. On the other hand, values for arteriovenous oxygen difference calculated from these data are suitable for detecting small errors, since slight inaccuracies in timing samples are not important in this connection. A definite but short plateau in the values for arteriovenous oxygen difference is evident before recirculation occurs, in this case some time between 14 and 18 seconds after the beginning of rebreathing. This plateau represents the true value for arteriovenous oxygen difference uninfluenced by errors due to lack of lung-bag equilibrium or recirculation of acetylene.

The time of this plateau is variable from person to person and in the same subject from day to day. Figure $2 A$ represents a continuous plateau with equilibration after 6 seconds and little or no recirculation at $20 \mathrm{sec}-$ onds. This result is no more accurate than the short but definite plateau in Figure 1. Figure $2 D$ illustrates the occurrence of recirculation without establishment of lung-bag equilibrium. If six respirations are made and samples of "alveolar" and "bag" gas are taken after the second and each succeeding expiration, and the rebreathing is completed in about 20 seconds, a plateau with vahues calculated from "alveolar" and "bag" samples coinciding is usually demonstrated. This ordinarily occurs between the third and fifth expirations and, when this is so, analyses of the second, third, and fourth samples from each source are sufficient. It is desirable to have the other samples available, however, because when irregular results are encountered in the calculations from these six samples a plateau may be demonstrated earlier or later from the other two pairs of samples. If not, the cause of the unsatisfactory result may often be identified and the next rebreathing modified by changing the sampling time or the volume of gas in the bag.

The determination can be accepted as accurate only when the values calculated from "bag" samples coincide with those calculated from "alveolar" samples, indicating lung-bag equilibrium during a plateau consisting of successive values in agreement, thus signifying the absence of the errors to be described below.

The principles used in evaluating each result are also illustrated in Figure 2. Some of the individual values in each graph have been disregarded as obviously due to errors described below. The range within which the arteriovenous oxygen difference can be said to lie, after the obvious errors have been discarded, is indicated in each graph by a vertical line at the left. When the range is small it is valid to accept the midpoint of that range as the result, subject to an error of half that range. In instances in which the range is larger it is safer to consider the range within which the value may possibly fall; acceptance of the most probable value within that range, as in Figure $2 B$, should wait upon additional determinations.

Sources of error. (1) Leaks. Very strenuous breathing, either inspiration or expiration, increases the incidence of mouthpiece and nose-clip leaks. Leaks during sampling may also occur. Irregularities in results caused by these factors are readily detected (Figure $2 B$ and $C$ ).

(2) Small lung-bag volume. Small total lung-bag volume, due either to small initial volume or to withdrawal of frequent or large samples, causes an error resembling that due to recirculation. This error increases progressively from interval to interval as the lung-bag volume is reduced, since the samples show progressively larger oxygen and acetylene percentages in relation to true alveolar gas, and since more acetylene than oxygen usually is absorbed. In practice, it is not always possible to distinguish between the effect of recirculation and this error.

(3) Inadequate mixing. Errors due to incomplete mixing are revealed by marked differences between " bag " and "alveolar" gas concentrations. Calculations of arteriovenous oxygen difference from data obtained before lung-bag equilibrium is attained, yield falsely high values if "alveolar" gas is used and falsely low values if "bag" gas is used (Figure 1). Errors introduced by lack of equilibrium of acetylene and oxygen are in opposite directions; since the lack of acetylene equilibrium is more marked at the beginning and more persistent, the total errors are in the direction of the errors due to acetylene imbalance.

(4) Recirculation. Errors due to this factor are readily detected by the absence of a definite plateau. This error can occur as early as $\mathbf{1 5}$ seconds after the beginning of rebreathing but in some instances there is no evidence of recirculation up to 20 seconds. Individuals show considerable day-to-day variation in this respect. When samples are taken after each expiration this error can be identified more accurately than with few samples. Samples from two sources help to distinguish errors due to recirculation from other errors.

(5) Analytical errors. Analytical inaccuracies introduce larger errors in the final result when sampling is frequent and the differences between successive samples are small; but if duplicate values agree within 0.03 per cent, the final error is seldom large enough to be important. Nevertheless, the accuracy of gas analyses is a factor which largely determines the accuracy of this method.

Accuracy of method. Eighty-two rebreathings have been performed on five subjects, using the method as de- 


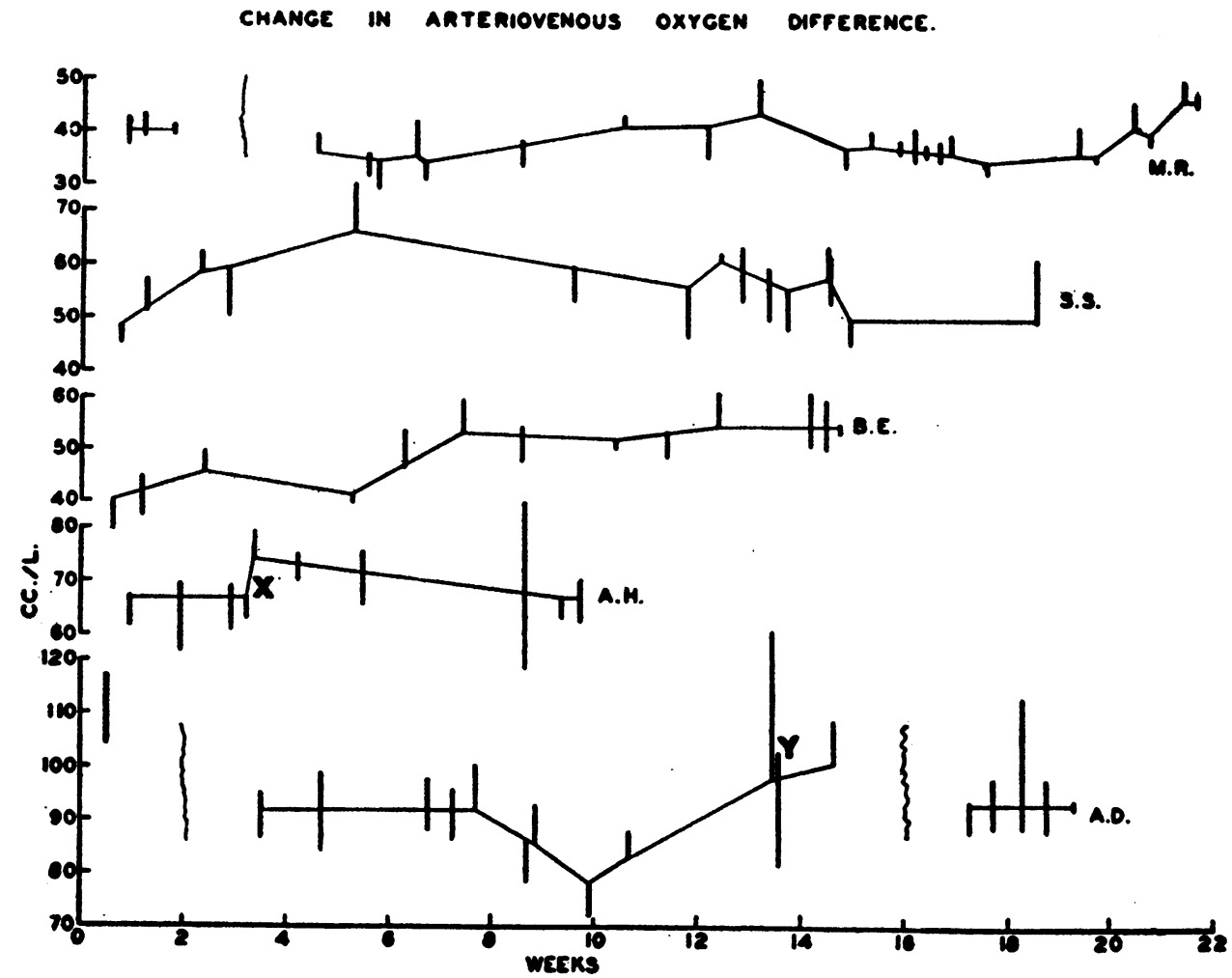

Fig. 3. The Vertical Bars Represent the Range of Arteriovenous Oxygen Difference Determined for Each Rebreathing as in Figure 2

The values are those included in Table I. The abscissa represents the time relationship of the determinations. The bars representing the determinations on each individual are connected by a line drawn as nearly horizontal as possible through the whole series. This indicates the minimum variation from time to time.

TABLE I

Range of accuracy of arteriovenous oxygen difference in 82 determinations on two normal subjects and three patients *

\begin{tabular}{|c|c|c|c|c|c|c|}
\hline \multirow{4}{*}{$\begin{array}{l}\text { Range (Per cent } \\
\text { of midpoint) }\end{array}$} & \multicolumn{6}{|c|}{ Number of determinations } \\
\hline & \multicolumn{5}{|c|}{ Subjects } & \multirow{3}{*}{$\begin{array}{l}\text { Total } \\
\text { sub- } \\
\text { jects }\end{array}$} \\
\hline & \multicolumn{2}{|c|}{ Normals } & \multicolumn{3}{|c|}{ Patients * } & \\
\hline & s. S. & B. E. & M. R. & A. $\mathbf{D}$. & A. $\mathbf{H}$. & \\
\hline $\begin{array}{r}0-5 \ldots \ldots \ldots \\
\mathbf{6}-10 \ldots \ldots \ldots \\
11-15 \ldots \ldots \ldots \\
16-20 \ldots \ldots \ldots \\
21+\ldots \ldots \ldots \ldots\end{array}$ & $\begin{array}{l}1 \\
3 \\
5 \\
5 \\
1\end{array}$ & $\begin{array}{l}3 \\
2 \\
4 \\
4\end{array}$ & $\begin{array}{r}4 \\
11 \\
8 \\
3\end{array}$ & $\begin{array}{l}1 \\
8 \\
5 \\
1 \\
3\end{array}$ & $\begin{array}{l}5 \\
2 \\
2 \\
1\end{array}$ & $\begin{array}{r}9 \\
29 \\
24 \\
15 \\
5\end{array}$ \\
\hline
\end{tabular}

* See text for diagnosis.

Subjects were sitting in bed with the back rest elevated to an angle of $45^{\circ}$.

scribed above. Subjects S. S. and B. E. were normal, subjects A. H. and A. D. had chronic rheumatic heart disease with symptoms of cardiac insufficiency but no râles in the lungs, and subject $M$. R. was convalescing from rheumatic fever with intermittent slight fever but no increase in sedimentation rate or leukocytosis; she had been in bed for over a year at the time of study. All determinations were made in the morning under basal conditions.

Maximum and minimum values for each rebreathing are indicated in Table $I$ and Figure 3, individual values obviously in error being discarded according to principles illustrated in Figure 2. No completed rebreathings are omitted, however. In Table I the difference between maximum and minimum possible values is expressed in percentage of the midpoint between them so that the greatest possible error of this midpoint is half the difference between the maximum and minimum values. In 38 of the 82 determinations the error was 5 per cent or less and in 62 of the 82 it was 7.5 per cent or less.

The accuracy with which physiologic variation can be determined is limited by the precision of individual values. The minimum real change in these subjects is represented graphically in Figure 3. The actual variation may exceed this. For example, the pair of determinations made on successive days on patient A. H. (marked X, Figure 
3) shows a definite minimum increase of $6.5 \mathrm{cc}$. per liter in arteriovenous oxygen difference represented by the line connecting the bars, and a maximum possible real increase of $15.3 \mathrm{cc}$. per liter (the difference between opposite ends of the bars). This change could be expressed as $10.9 \pm 4.4$ cc. per liter.

At times 2 determinations, each of which is unsatisfactory, may provide reasonably good evidence that the arteriovenous oxygen difference lies within a narrow range as shown in the two observations marked $Y$ (Figure 3, patient A. D.).

\section{DISCUSSION}

The studies described here demonstrate that complete mixing in the lung-bag system used may occur in two breaths and is usually achieved by four breaths. This is earlier than was indicated by the experiments of Grollman and Marshall (4) who concluded that equilibration required $15 \mathrm{sec}-$ onds. Gladstone and Dack (5), however, have pointed out the fallacy in the work of Grollman and Marshall. The present studies confirm the observations of Gladstone and Dack in regard to the excretion of excessive amounts of $\mathrm{CO}_{2}$ during the first 15 seconds of rebreathing; lung-bag equilibrium could not become apparent in Grollman and Marshall's experiments (4) while an excess of $\mathrm{CO}_{2}$ was being removed from the blood by deep breathing. The contention of Grollman and Marshall that rebreathing must proceed for 15 seconds before lung-bag equilibration is accomplished is therefore apparently erroneous. The modified method described here makes possible the certain detection of inadequate lung-bag mixing and so avoids the errors pointed out by Grollman (1) and Hamilton, Spradlin, and Saam (3).

That recirculation of blood containing acetylene occurs in less than 20 seconds has been shown by Hamilton et al. (3), Starr and Collins (6) and Gladstone (7), as well as by the results of the experiments described here. However, the modified method suggested obviates errors due to this factor.

Although the modifications suggested here make the method more involved, it is less complicated for the subject than partial inspiration from each of two bags, as in the Gladstone modification (8). Reduction of the volume of the lung-bag system, as recommended by McMichael (9), may serve to increase rather than decrease the errors of the original Grollman method. Time expended in making gas analyses is increased two- to fourfold by our method but this increase is made worthwhile by considerations outlined above.

While this method makes possible the recognition of errors due to incomplete lung-bag equilibrium, recirculation, leaks in the lung-bag system and sampling tubes and analytical inaccuracies, it should be pointed out that there are other uncertainties in the method. Some of these are: incomplete saturation of blood by acetylene due to circulatory shunts from one side of the heart to the other and to abnormalities of the lung; changes of alveolar oxygen concentration by deep breathing when alveolar oxygen falls too low during the determination or when the arterial oxygen saturation is below normal during normal respiration (the alveolar oxygen, and therefore the arterial saturation, are raised during rebreathing); changes in the blood which modify the solubility coefficient of acetylene; and diffusion of acetylene into tissues of the respiratory tract other than blood. These errors are probably small or of only occasional occurrence and have been discussed elsewhere.

\section{CONCLUSIONS}

1. In determining arteriovenous oxygen difference with the acetylene method, multiple samples of "alveolar" and "bag" gas make it possible to identify the establishment of lung-bag equilibrium of the acetylene-air mixture and the beginning of recirculation of acetylene-containing blood to the lungs in each instance. Analytical inaccuracies and leaks, as well as most of the many irregularities encountered in a procedure of this type, are identified.

2. The time required to achieve lung-bag equilibrium and the time of the first appearance of recirculation are variable, but they usually occur much earlier in the period of rebreathing than Grollman (2) indicated.

3. The interval between the establishment of equilibrium and the occurrence of recirculation varies, but is usually long enough to allow at least two complete respirations. This is sufficient for an accurate determination of arteriovenous oxygen difference.

4. Seventy-six per cent of 82 determinations were in error by less than 7.5 per cent. 


\section{BIBLIOGRAPHY}

1. Marshall, E. K., Jr., and Grollman, A., A method for the determination of the circulatory minute volume in man. Am. J. Physiol., 1928, 86, 117.

2. Grollman, A., The Cardiac Output of Man in Health and Disease. Charles C. Thomas, Springfield, 1932.

3. Hamilton, W. F., Spradlin, M. C., and Saam, H. G., $\mathrm{Jr}$., An inquiry into the basis of the acetylene method of determining the cardiac output. Am. J. Physiol., 1932, 100, 587.

4. Grollman, A., and Marshall, E. K., Jr., The time necessary for rebreathing in a lung-bag system to attain homogeneous mixture. Am. J. Physiol., 1928, 86, 110.

5. Gladstone, S. A., and Dack, S., Respiratory exchange of oxygen and carbon dioxide during rebreathing from a rubber bag. Proc. Soc. Exper. Biol. and Med., 1935, 32, 1315.

6. Starr, I., Jr., and Collins, L. H., Jr., Estimations of the rapidity and amount of blood traversing the shorter paths of the systemic circulation. Am. J. Physiol., 1933, 104, 650.

7. Gladstone, S. A., Cardiac Output and Arterial Hypertension. Privately Published. New York City, 1935.

8. Gladstone, S. A., A modified foreign-gas method for determination of cardiac output in man. Proc. Soc. Exper. Biol. and Med., 1935, 32, 1321.

9. McMichael, J., Postural changes in cardiac output and respiration in man. Quart. J. Exper. Physiol., 1937, 27, 55. 